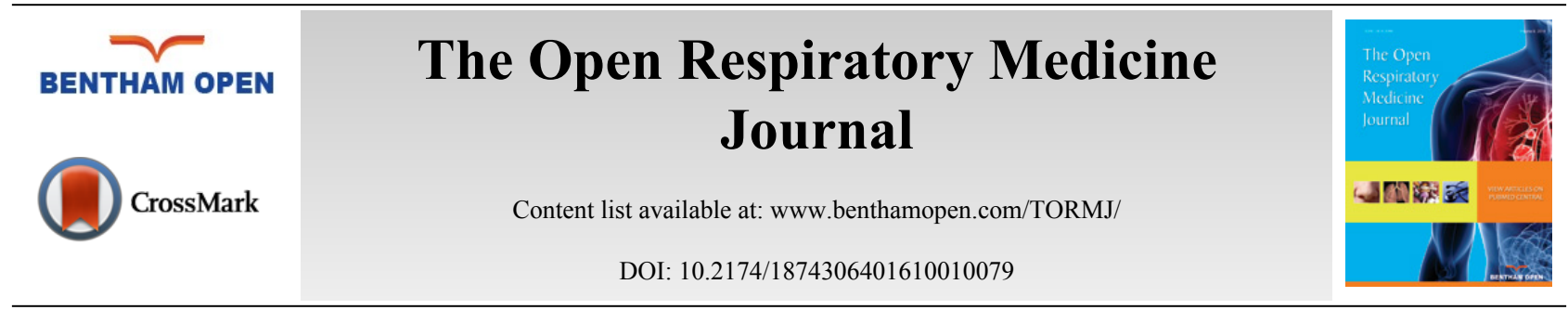

RESEARCH ARTICLE

\title{
Evaluation of Pulmonary Nodules Using the Spyglass Direct Visualization System Combined With Radial Endobronchial Ultrasound: A Clinical Feasibility Study
}

\author{
Krystelle Godbout, Simon Martel, Mathieu Simon, Noël Lampron and Antoine Delage* \\ Département Multidisciplinaire de Pneumologie et Chirurgie Thoracique, Institut Universitaire de Cardiologie et de \\ Pneumologie de Québec, Québec, Québec, Canada
}

Received: June 28, 2016

Revised: November 10, 2016

Accepted: November 14, 2016

\begin{abstract}
:
Background:

Sampling of peripheral pulmonary nodules with radial endobronchial ultrasound (p-EBUS) increases diagnostic yield of bronchoscopy. However, diagnostic yield is influenced by numerous factors.
\end{abstract}

\section{Objective:}

We evaluated the use of SpyGlass, a one millimeter diameter optic fiber, to obtain images of the distal mucosa and of pulmonary lesions detected with p-EBUS to determine if visual aspect of the distal mucosa was predictive of diagnosis.

\section{Methods:}

We prospectively recruited subjects investigated for peripheral nodules. Bronchoscopy was performed and p-EBUS was used to locate the lesion through a guide sheath. The Spyglass fiber was introduced in the sheath to obtain images of the distal bronchial mucosa. Tissue sampling was subsequently done.

\section{Results:}

Fifteen patients were enrolled in the study. A final diagnosis of malignancy was confirmed in $80 \%$. All lesions could be located using p-EBUS (100\%). Diagnostic sensitivity for p-EBUS was 58.3\%. Distal mucosa could be imaged with SpyGlass in 14/15 patients (93.3\%). Mucosal appearance was described as abnormal in 7 out of the 15 subjects. Mean SpyGlass procedure time was 6.5 minutes. No direct complication was reported.

\section{Conclusion:}

Spyglass can be used in combination with p-EBUS to obtain images of the distal bronchial mucosa and peripheral pulmonary nodules. More patients will be needed to confirm whether mucosal appearance can be predictive of malignancy.

Keywords: Bronchoscopy, Endobronchial ultrasound, Lung cancer, Optic fiber, Peripheral nodule.

\section{INTRODUCTION}

The need to investigate peripheral lung nodule is a commonly encountered clinical situation in pulmonary medicine. Histological diagnosis of such lesions is crucial to prevent unnecessary surgeries for benign lesions and to orient therapy in cases of malignancy. Standard bronchoscopy has a diagnostic yield limited to $10-50 \%$ for peripheral

\footnotetext{
* Address correspondence to this author at the Département Multidisciplinaire de Pneumologie et Chirurgie Thoracique, Institut Universitaire de Cardiologie et de Pneumologie de Québec, 2725 Chemin Ste-Foy, Québec, Québec, Canada, G1V 4G5; Tel: +1-418-656-4747; Fax: +1-418-656-4762; E-mail: antoine.delage@criucpq.ulaval.ca
} 
pulmonary lesions less than $2 \mathrm{~cm}$ diameter [1]. Radial endobronchial ultrasound (p-EBUS) improves the diagnostic yield of bronchoscopy for peripheral lung nodules. Using a small ultrasound probe, the peripheral nodule is located and then sampled through a guide sheath. Studies have shown diagnostic yields of $58-84 \%$ with p-EBUS. Positive results correlated with size of the nodule, presence of a "bronchus sign" on computer tomography, position of the probe with respect to the lesion and sampling methods [2 - 13]. A meta-analysis of the use of p-EBUS showed a pooled sensitivity of $73 \%$ for diagnosis of cancer using this technique. However there was a significant heterogeneity in study populations [14]. The use of fine needle aspiration has been shown to increase the diagnostic yield of p-EBUS. This could be explained by the fact that the lesion is located behind normal bronchiolar mucosa at the distal end of the guide sheath [15]. Hence, the ability to visualize the aspect of the mucosa at the distal end of the guide sheath prior to sampling could prove useful in choosing sampling methods and eventually improve diagnostic sensitivity for pEBUS.

The Spyglass Direct Vizualization System (Boston Scientific, Natick, MA, USA) is a 0.9 mm diameter optic fibre probe developed for the evaluation of biliary tree (Fig. 1). It has been shown to provide valuable images of the bile ducts [16]. The use of this system in the bronchial tree has not been described but appears technically feasible. In combination with p-EBUS, it could be used to visualize the distal bronchus through the catheter guide sheath to predict sampling results and eventually guide choices in sampling techniques.

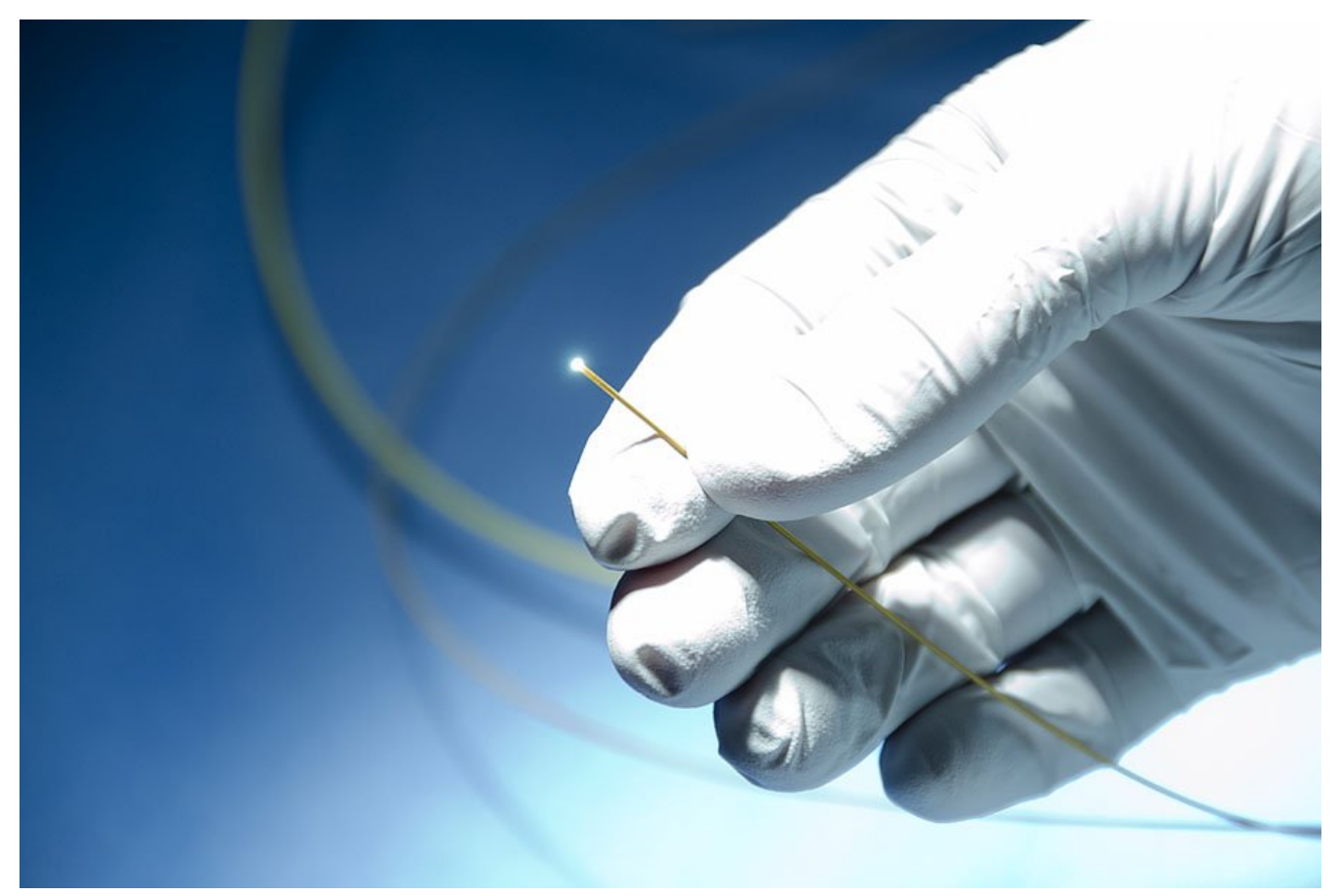

Fig. (1). SpyGlass optic Fiber has a diameter of $0.9 \mathrm{~mm}$.

This study aimed to evaluate if the distal bronchial tree and peripheral pulmonary lesions could be imaged using the Spyglass optic fiber probe by inserting it through the bronchoscopic guide sheath after nodule detection with p-EBUS. We also wanted to verify if the aspect of the observed bronchial mucosa was predictive of diagnosis.

\section{MATERIALS AND METHODS}

\section{Population}

Fifteen subjects were recruited at our institution to participate in the study. Subjects were consecutive patients who underwent diagnostic bronchoscopy with p-EBUS for the evaluation of a peripheral pulmonary nodule of more than 10 $\mathrm{mm}$ in diameter. All study subjects were first evaluated by one of the pulmonary specialists from our institution who planned the pEBUS procedure. Computed axial tomography imaging of the lung parenchyma was available for all study subjects. All imaging was reviewed by the physician performing the bronchoscopy and p-EBUS prior to inclusion in the study. Size of the lesion on CT scan was measured using its longest diameter. Peripheral pulmonary lesion was defined as a lesion completely surrounded by pulmonary parenchyma and not visible on bronchoscopy. 
The study protocol was approved by the institutional review board. Signed informed consent was obtained from all patients prior to inclusion in the study.

\section{Procedure}

The procedures were performed by 4 bronchoscopists (AD, SM, MS, NL) with proficiency for p-EBUS. A second physician experienced in p-EBUS was present to assist during the Spyglass procedure. The main study investigator (AD) was present for all study procedures. All procedures were performed under conscious sedation using a combination of intravenous midazolam and fentanyl. A therapeutic videobronchoscope was used (BF-T180 and BFT160, Olympus, Tokyo, Japan).

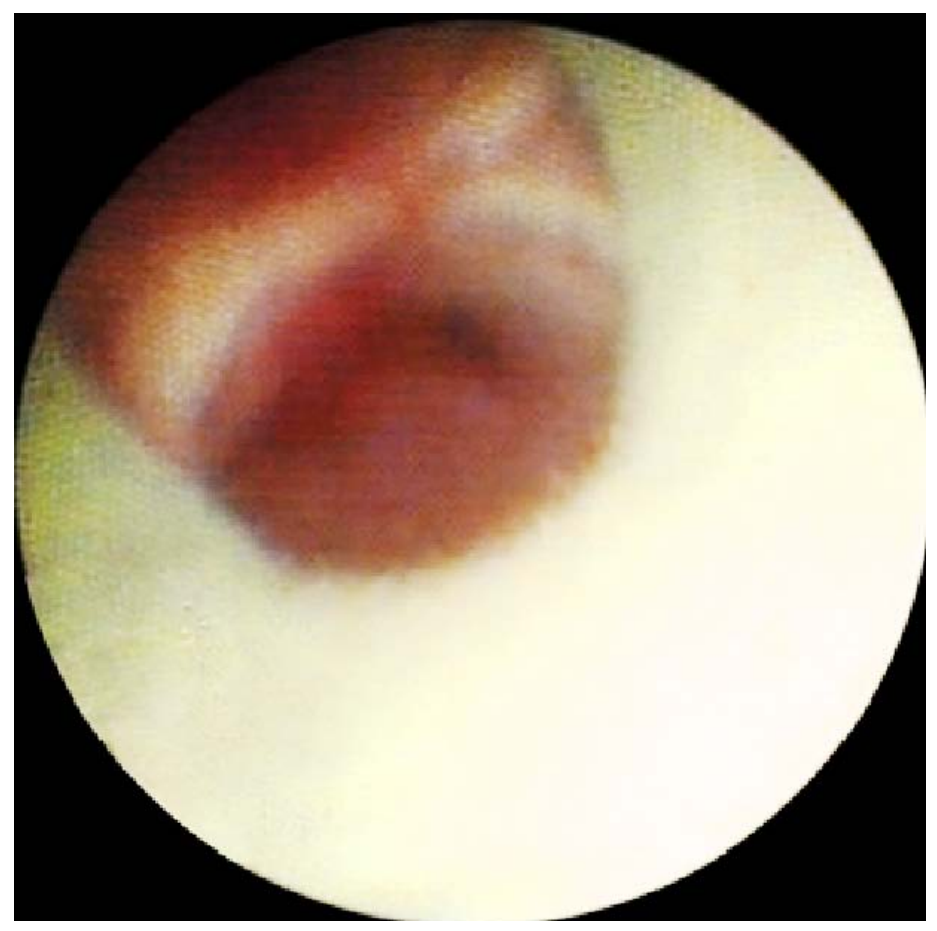

Fig. (2). Spyglass image of normal appearing carina distinguished at the end of the guide sheath (which appears yellow in the lower part of the picture).

If no lesion suspect was identified after standard bronchoscopy, p-EBUS was performed using Olympus endoscopic ultrasound processor (UM-4R, Olympus, Tokyo, Japan) with a $1.7 \mathrm{~mm}$ diameter flexible radial probe (UM-S20-20R, Olympus, Tokyo, Japan) inserted through a $2.7 \mathrm{~mm}$ diameter guide sheath (XB01-836-13, Olympus, Tokyo, Japan) (GS). The GS-covered probe was then used to localize the lesion. Once the lesion was located, an ultrasound image was recorded and the probe removed with the GS let in place.

Subsequently, the SpyGlass optic fiber (Boston Scientific, Natick, MA, USA) (Fig. 1) was inserted through the GS and advanced at the distal end of the sheath. The SpyGlass probe has an external diameter of 0.9 mm and a length of $231 \mathrm{~cm}$ (Fig. 1). It contains a 6,000 pixel image bundle surrounded by approximately 225 light transmission fibers. The lens at the tip of the optic fiber provides a $70^{\circ}$ field of view. Physiologic serum was instilled ( $10 \mathrm{ml}$ at a time up to a maximum of $150 \mathrm{ml}$ ) concurrently through the GS using a distal sideport in order to open the small distal airways and achieve the best image possible. Total amount of fluid instilled was recorded. Images of the p-EBUS and Spyglass examination were recorded on a video disc for subsequent review. If the distal mucosa could be imaged, the physician performing the procedure reported if the mucosa appeared normal or abnormal. To determine the appearance of normal mucosa, during the first three cases that were done, the probe was inserted in a separate, healthy appearing part of the lung and images subsequently obtained with the optic fiber. The aspect of the mucosa was defined as normal when a carina was found and of normal appearance after viewing the first three cases as described above Fig. (2). After images were obtained, the SpyGlass optic fiber was removed. Total time to perform the Spyglass bronchial visualization was recorded. Thereafter, p-EBUS probe was reintroduced in the guide sheath to ascertain proper positioning within the 
lesion prior to sampling.

Sampling of the lesion was then performed through the sheath in the following sequence: five transbronchial forceps biopsies, fine needle aspiration (not done in some subjects because the needle would not pass through a bended catheter), cytology brush and bronchoalveolar lavage using three $50 \mathrm{ml}$ aliquots of normal saline at body temperature. A chest X-ray was performed following procedure to detect pneumothorax. Clinically significant bleeding that required intervention was recorded as well. Follow-up data was obtained in all patients to ascertain final diagnosis.

\section{RESULTS}

Fifteen patients were enrolled in the study. Characteristics are shown in Table $\mathbf{1 .}$

All lesions were located using p-EBUS (100\%). Position of the probe with respect to the lesion was central in $60 \%$ (9 patients) and peripheral in $40 \%$ (6 subjects).

A definitive diagnosis could be obtained in 14 subjects. One subject did not have a definitive pathological diagnosis but proved to have a benign course with complete radiological regression of the lesion at 6 months follow-up. Malignancy was the final diagnosis in 12 patients (80\%): 11 patients with a primary pulmonary neoplasia and 1 with a metastatic melanoma. The two confirmed benign cases were proven by subsequent open lung resection and consisted in one case of fungal infection and one case of fibro-inflammatory remodeling.

Using the SpyGlass system, images of the distal mucosa could be obtained in 14/15 patients. Extrinsic compression of the distal end of the guide sheath prevented passage of the optic fiber and visualization the distal mucosa in one patient. Mucosal aspect was described as normal in 50\% of cases (7/14) and abnormal in the other 50\% (Figs. 2 and 3 ).

Characteristics and results for each individual subject are shown in Table 1. p-EBUS samplings were diagnostic in $7 / 15$ of cases $(46 \%)$.

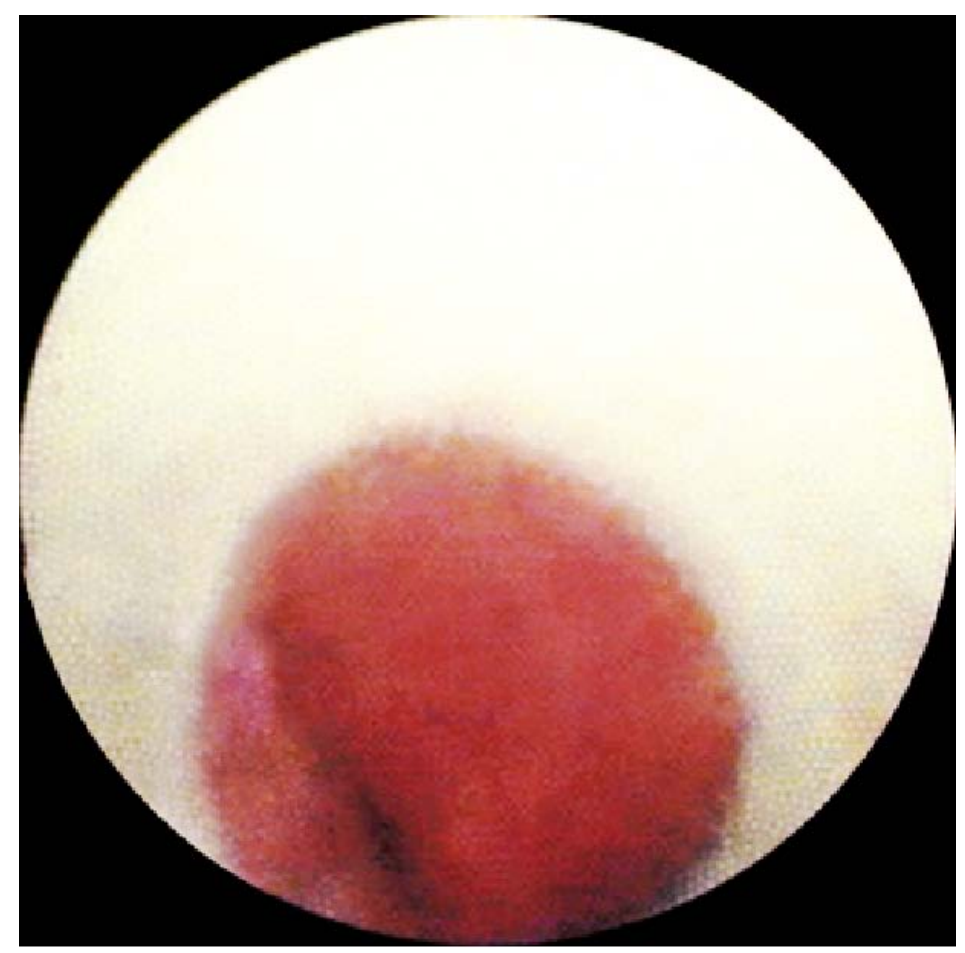

Fig. (3). Spyglass image: Infiltrated aspect of the mucosa with narrowing of the bronchial lumen is seen at the end of the guide sheath. Transbronchial biopsy and cytology brushings confirmed adenocarcinoma of the lung.

Procedure prolongation time for SpyGlass was 6 minutes 30 seconds on average (3-12 minutes). The last four Spyglass procedures all lasted less than 5 minutes.

No direct complication of SpyGlass was reported. One pneumothorax occurred after trans-bronchial biopsies and did not require drainage. It was attributed to the trans-bronchial biopsy procedure itself rather than the Spyglass examination. No bleeding was reported. 
Table 1. Patient characteristics and results with corresponding Spyglass optic fiber appearance.

\begin{tabular}{|c|c|c|c|c|c|c|c|c|}
\hline ID & Sex & Age & $\begin{array}{c}\text { Lesion size } \\
(\mathbf{m m})\end{array}$ & Localisation & p-EBUS position & SpyGlass appearance & Sampling results & Final diagnosis \\
\hline $\mathbf{1}$ & M & 77 & 38 & RUL & Central & Abnormal (infiltrated) & Adenocarcinoma & Adenocarcinoma \\
\hline $\mathbf{2}$ & M & 53 & 18 & LLL & Central & Abnormal (mucus) & Non-diagnostic & Infection \\
\hline $\mathbf{3}$ & F & 50 & 32 & LLL & Adjacent & Abnormal (granulation) & Non-diagnostic & Adenocarcinoma \\
\hline 4 & F & 48 & 52 & RUL & Central & Normal & Granuloma & Fungal infection \\
\hline $\mathbf{5}$ & F & 66 & 35 & RLL & Central & Normal & Adenocarcinoma & Adenocarcinoma \\
\hline $\mathbf{6}$ & F & 63 & 18 & RUL & Adjacent & Normal & Non-diagnostic & Fibrosis \\
\hline $\mathbf{7}$ & M & 79 & 42 & RML & Adjacent & Normal & Non-diagnostic & Squamous cell carcinoma \\
\hline $\mathbf{8}$ & F & 50 & 21 & LUL & Adjacent & Technically impossible & Non-diagnostic & Metastatic melanoma \\
\hline $\mathbf{9}$ & F & 68 & 54 & RUL & Central & Normal & Non-diagnostic & Bronchioloalveolar carcinoma \\
\hline $\mathbf{1 0}$ & M & 65 & 25 & RUL & Adjacent & Normal & Adenocarcinoma & Adenocarcinoma \\
\hline $\mathbf{1 1}$ & M & 63 & 27 & RUL & Adjacent & Abnormal (hemorrhagic) & Adenocarcinoma & Adenocarcinoma \\
\hline $\mathbf{1 2}$ & F & 59 & 46 & LUL & Central & Abnormal (infiltrated) & Adenocarcinoma & Adenocarcinoma \\
\hline $\mathbf{1 3}$ & M & 68 & 26 & RUL & Central & Abnormal (granulation) & Non-diagnostic & Squamous cell carcinoma \\
\hline $\mathbf{1 4}$ & M & 68 & 23 & RUL & Central & Normal & Adenocarcinoma & Adenocarcinoma \\
\hline $\mathbf{1 5}$ & M & 61 & 74 & RUL & Central & Abnormal (infiltrated) & NSCLC & NSCLC \\
\hline
\end{tabular}

\section{DISCUSSION}

Standard flexible bronchoscopy allows only the first 5 or 6 generations of the bronchial tree to be examined using a flexible bronchoscope, thus limiting its usefulness in the evaluation of peripheral pulmonary lesions. The use of radial probe EBUS has been shown to increase the diagnostic yield of bronchoscopy for peripheral lesions to 73\% [14]. However, in our experience, biopsy specimens often turn out to be non-diagnostic despite ascertained localization of pulmonary nodules with the p-EBUS. In one study, performing fine needle aspiration through the p-EBUS guide sheath in addition to transbronchial biopsy significantly increased the diagnostic yield of bronchoscopy with p-EBUS [15]. This finding could be explained by the fact that the abnormal or malignant tissue lies beyond the airway wall. Hence, being able to visualize the mucosa at the end of the guide sheath could possibly help guide sampling methods for $\mathrm{p}$ EBUS. In this feasibility study, we hypothesized that the Spyglass Direct Visualization system, a $0.9 \mathrm{~mm}$ optic fiber developed for diagnostic examination the biliary tree, could be used to obtain images of the distal bronchial mucosa and pulmonary nodules.

We performed 15 Spyglass procedures after nodule localization with p-EBUS. Images of the distal mucosa could be obtained in all but one subject. We also were able to distinguish normal from abnormal bronchial mucosa. The Spyglass procedure added on average 6 minutes and 30 seconds to the entire procedure. Procedure time decreased with increasing number of procedures, suggesting there may be a learning curve for the personnel and physician performing it. Proper positioning of the optic fiber within the guide sheath and opening of the distal airway with small amounts of instilled saline were necessary to obtain clear and interpretable images of the distal mucosa.

This study aimed at determining whether the Spyglass optic fiber could be used to image the distal airways. It was not powered to evaluate the diagnostic value of optic visualization of the distal mucosal appearance. However, in our series, mucosal aspect seemed to be predictive of a cancer diagnosis. More subjects will be needed to confirm this hypothesis.

In the present study, we observed that diagnostic yield and sensitivity for p-EBUS are lower than those reported in the literature [2 - 14]. We asked ourselves whether manipulations associated with the SpyGlass could have displaced the guide sheath prior to sampling. However, in all cases, adequate positioning of the sheath was ascertained by repeating the p-EBUS after the Spyglass and prior to sampling. Hence, this diagnostic gap is more likely to be due to sampling techniques and subject-related factors. Also, the small number of patients studied makes these results unreliable as one or two additional positive or negative cases could significantly influence sensitivity and diagnostic accuracy.

In conclusion, we demonstrated the feasibility of using the SpyGlass Direct Visualization System optic fiber via a guide sheath to obtain images of the distal bronchial mucosa. Given the small diameter of this fiber, it allowed us image the bronchial mucosa farther than the smallest available bronchoscope would have likely allowed. More subjects will be needed to confirm whether aspect of the mucosa could predict a positive pEBUS diagnosis. It is possible that 
visualizing the aspect of the distal mucosa could orient the bronchoscopist in choosing subsequent sampling procedures, notably by adding needle aspiration, and help increase the diagnostic yield for this procedure. As technology improves, the day might come when the endoscopist will be able to directly locate and visualize peripheral pulmonary nodules, thus increasing the diagnostic yield of bronchoscopy for this condition.

\section{AUTHOR CONTRIBUTION}

Dr. Krystelle Godbout participated in study planning, data analysis and was the primary author of the manuscript.

Dr. Simon Martel participated in study planning, execution, editing of the manuscript and was co-primary investigator of the study.

Dr. Mathieu Simon participated in study planning, execution, and editing of the manuscript.

Dr. Noël Lampron participated in study planning, execution, and editing of the manuscript.

Dr. Antoine Delage participated in study planning, execution, data analysis, editing of the manuscript and was coprimary investigator of the study.

\section{FUNDING}

Study funding was obtained through an unrestricted grant from the Fondation de l'Institut Universitaire de Cardiologie et Pneumologie de Québec. Boston Scientific did not finance the study or participate in its execution.

\section{CONFLICT OF INTEREST}

The authors confirm that this article content has no conflict of interest.

\section{ACKNOWLEDGEMENTS}

The authors would like to thank Dr Michel Laviolette for the help provided during this study.

\section{REFERENCES}

[1] Gould MK, Fletcher J, Iannettoni MD, et al. Evaluation of patients with pulmonary nodules: when is it lyng cancer? ACCP evidence-based clinical practice guidelines (2nd edition) Chest 2007; 132: 108S-30S.

[2] Herth FJ, Ernst A, Becker HD. Endobronchial ultrasound-guided transbronchial lung biopsy in solitary pulmonary nodules and peripheral lesions. Eur Respir J 2002; 20(4): 972-4.

[http://dx.doi.org/10.1183/09031936.02.00032001] [PMID: 12412691]

[3] Kurimoto N, Miyazawa T, Okimasa S, et al. Endobronchial ultrasonography using a guide sheath increases the ability to diagnose peripheral pulmonary lesions endoscopically. Chest 2004; 126(3): 959-65. [http://dx.doi.org/10.1378/chest.126.3.959] [PMID: 15364779]

[4] Kikuchi E, Yamazaki K, Sukoh N, et al. Endobronchial ultrasonography with guide-sheath for peripheral pulmonary lesions. Eur Respir J 2004; 24(4): 533-7.

[http://dx.doi.org/10.1183/09031936.04.00138603] [PMID: 15459129]

[5] Paone G, Nicastri E, Lucantoni G, et al. Endobronchial ultrasound-driven biopsy in the diagnosis of peripheral lung lesions. Chest 2005; 128(5): 3551-7.

[http://dx.doi.org/10.1378/chest.128.5.3551] [PMID: 16304312]

[6] Herth FJ, Eberhardt R, Becker HD, Ernst A. Endobronchial ultrasound-guided transbronchial lung biopsy in fluoroscopically invisible solitary pulmonary nodules: a prospective trial. Chest 2006; 129(1): 147-50. [http://dx.doi.org/10.1378/chest.129.1.147] [PMID: 16424425]

[7] Eberhardt R, Anantham D, Ernst A, Feller-Kopman D, Herth F. Multimodality bronchoscopic diagnosis of peripheral lung lesions: a randomized controlled trial. Am J Respir Crit Care Med 2007; 176(1): 36-41.

[http://dx.doi.org/10.1164/rccm.200612-1866OC] [PMID: 17379850]

[8] Yoshikawa M, Sukoh N, Yamazaki K, et al. Diagnostic value of endobronchial ultrasonography with a guide sheath for peripheral pulmonary lesions without X-ray fluoroscopy. Chest 2007; 131(6): 1788-93. [http://dx.doi.org/10.1378/chest.06-2506] [PMID: 17565021]

[9] Yamada N, Yamazaki K, Kurimoto N, et al. Factors related to diagnostic yield of transbronchial biopsy using endobronchial ultrasonography with a guide sheath in small peripheral pulmonary lesions. Chest 2007; 132(2): 603-8. [http://dx.doi.org/10.1378/chest.07-0637] [PMID: 17573504]

[10] Chung YH, Lie CH, Chao TY, et al. Endobronchial ultrasonography with distance for peripheral pulmonary lesions. Respir Med 2007; 101(4): 738-45. 
[http://dx.doi.org/10.1016/j.rmed.2006.08.014] [PMID: 17015004]

[11] Asano F, Matsuno Y, Tsuzuku A, et al. Diagnosis of peripheral pulmonary lesions using a bronchoscope insertion guidance system combined with endobronchial ultrasonography with a guide sheath. Lung Cancer 2008; 60(3): 366-73. [http://dx.doi.org/10.1016/j.lungcan.2007.10.022] [PMID: 18055063]

[12] Fielding DI, Robinson PJ, Kurimoto N. Biopsy site selection for endobronchial ultrasound guide-sheath transbronchial biopsy of peripheral lung lesions. Intern Med J 2008; 38(2): 77-84.

[http://dx.doi.org/10.1111/j.1445-5994.2007.01473.x] [PMID: 17916171]

[13] Mizugaki H, Shinagawa N, Kanegae K, et al. Combining transbronchial biopsy using endobronchial ultrasonography with a guide sheath and positron emission tomography for the diagnosis of small peripheral pulmonary lesions. Lung Cancer 2010; 68(2): 211-5. [http://dx.doi.org/10.1016/j.lungcan.2009.06.004] [PMID: 19595471]

[14] Steinfort DP, Khor YH, Manser RL, Irving LB. Radial probe endobronchial ultrasound for the diagnosis of peripheral lung cancer: systematic review and meta-analysis. Eur Respir J 2011; 37(4): 902-10.

[http://dx.doi.org/10.1183/09031936.00075310] [PMID: 20693253]

[15] Chao TY, Chien MT, Lie CH, Chung YH, Wang JL, Lin MC. Endobronchial ultrasonography-guided transbronchial needle aspiration increases the diagnostic yield of peripheral pulmonary lesions: a randomized trial. Chest 2009; 136(1): 229-36. [http://dx.doi.org/10.1378/chest.08-0577] [PMID: 18812446]

[16] Chen YK, Pleskow DK. SpyGlass single-operator peroral cholangiopancreatoscopy system for the diagnosis and therapy of bile-duct disorders: a clinical feasibility study (with video). Gastrointest Endosc 2007; 65(6): 832-41. [http://dx.doi.org/10.1016/j.gie.2007.01.025] [PMID: 17466202]

C) Godbout et al.; Licensee Bentham Open

This is an open access article licensed under the terms of the Creative Commons Attribution-Non-Commercial 4.0 International Public License (CC BY-NC 4.0) (https://creativecommons.org/licenses/by-nc/4.0/legalcode), which permits unrestricted, non-commercial use, distribution and reproduction in any medium, provided the work is properly cited. 